\title{
miRNA-375 regulates the cell survival and apoptosis of human non-small cell carcinoma by targeting HER2
}

\author{
LONGQIANG CHENG, BINGXIANG ZHAN, PENG LUO and BAOLONG WANG \\ Department of Clinical Laboratory, Affiliated Provincial Hospital of Anhui Medical University, \\ Hefei, Anhui 230001, P.R. China
}

Received September 20, 2015; Accepted October 31, 2016

DOI: $10.3892 / \mathrm{mmr} .2017 .6112$

\begin{abstract}
RNAs are a class of small non-coding RNAs that regulate gene expression by binding to the 3'-untranslated region of mRNA, which may lead to mRNA degradation or transcription regulation. Previous studies indicated that miRNAs are important for the pathogenesis of human cancer. miR-375 has been implicated in various tumor types; however, the biological activity in human non-small cell lung carcinoma (NSCLC) cells remains to be fully elucidated. The purpose of the present study was to investigate the biological importance of miR-375 in human NSCLC cells. The expression of miRNAs and mRNA was determined using reverse transcription-quantitative polymerase chain reaction. Cell proliferation was analyzed using a Cell Counting kit-8 assay. Cell apoptosis was analyzed using a fluorescence-activated cell sorting assay. The migration and invasion abilities of cells were evaluated using an in vivo mouse model. Dual-luciferase assay and western blotting were used to determine the potential target of miR-375. The results indicated that the expression of miR-375 in human NSCLC cells was significantly downregulated and induction of miR-375 may inhibit the proliferation of human NSCLC cells by inducing apoptosis. An animal model was used to determine that the upregulation of miR-375 inhibited the migration and invasion of A549 human NSCLC cells in vivo. It was also determined that human epidermal growth factor receptor 2 (HER-2) was a direct target gene of miR-375 and induction of miR-375 may reduce the expression of HER-2 in human NSCLC cells. These findings suggested that miR-375 may act as a potential therapeutic target for human NSCLC cancer in the future.
\end{abstract}

Correspondence to: Dr Baolong Wang, Department of Clinical Laboratory, Affiliated Provincial Hospital of Anhui Medical University, 17 Lujiang Road, Hefei, Anhui 230001, P.R. China E-mail: baolongw@yeah.net

Key words: miR-375, non-small cell lung cancer cell, proliferation, apoptosis, HER2

\section{Introduction}

Lung cancer is a malignant lung tumor characterized by uncontrolled tumor cell growth in the lungs (1) and has a high mortality rate worldwide. There are two main subtypes of lung cancer, non-small cell lung carcinoma (NSCLC) and small-cell lung carcinoma; $>80 \%$ of lung cancer is diagnosed as NSCLC (1). There are three subtypes of NSCLC: Adenocarcinoma, squamous-cell carcinoma and large-cell carcinoma (1). The majority of patients with NSCLC are diagnosed at an advanced stage, therefore, the mortality rate for lung cancer is high (2). The $>5$ year survival rate of patients with NSCLC is $\sim 11 \%$ despite improvement of the clinical treatments available (2). Current treatments for lung cancer, include surgery, radiotherapy and chemotherapy; however, the therapeutic effect is not satisfactory. Targeted therapy is a novel strategy used to treat lung cancer. Previous studies have demonstrated that micro (mi)-RNAs may be involved in the development of human NSCLC (3-5), which may provide a novel direction for the therapy of human NSCLC.

miRNAs are a series of small non-coding RNAs. They are 21-24 nucleotides long and regulate gene expression by binding to the 3'-untranslated region of target mRNAs $(6,7)$. The binding subsequently leads to mRNA degradation or transcriptional regulation $(8,9)$. At present $>700$ miRNAs have been identified in humans (miRBase Database, University of Manchester, UK). It is estimated that $>30 \%$ of human genes are regulated by miRNAs and that one miRNA may have several transcription targets. Abnormal expression of miRNAs may induce the dysregulation of genes, which may lead to the dysregulation of cell proliferation and cell apoptosis $(10,11)$. Previous studies indicated that abnormal expression of miRNAs was associated with the progression of cancer by the regulation of the oncogenes or tumor suppressors (12-16). Liu et al (5) indicated that downregulation of miRNA (miR)-10b may inhibit the proliferation and invasion of NSCLC cells (5). Liu et al (3) revealed that miR-196a promoted NSCLC cell proliferation and invasion by targeting homeobox A5 (3). Ma et al (4) determined that miR-143 inhibits migration and invasion of human NSCLC (4). Li et al (17) also reported that the expression of miR-375 was significantly reduced in human NSCLC tissues (17); however, the activity of miR-375 in human NSCLC cells remains to be fully elucidated. Additionally, 
the potential mechanism of miR-375 in human NSCLC cells requires investigation.

The present study focused on the expression of miR-375 in human NSCLC cells, in order to investigate the activity of miR-375 and to determine the potential target of miR-375 in human NSCLC cells.

\section{Materials and methods}

Animals. A total of $18 \mathrm{BALB} / \mathrm{c}$ nude male mice (4-6 weeks old) were purchased from Shanghai Laboratory Animal Center (Shanghai, China) and maintained under specific pathogen-free conditions at $24 \pm 2{ }^{\circ} \mathrm{C}$ and $50 \%$ humidity, and with a 12-h light/dark cycle. They were provided with sterilized food and water. All animal studies were in accordance with the Guide for the Care and Use of Laboratory Animals and were performed according to the ethical guidelines of Affiliated Provincial Hospital of Anhui Medical University (Anhui, China) for animal experiments. The present study was approved by the Ethics Committee of the Affiliated Provincial Hospital of Anhui Medical University (Hefei, China).

Cell culture. NSCLC adenocarcinoma cell lines A549, SPC-A-1, NCI-H1650, NCI-H1299, NSCLC squamous carcinoma cell line SK-MES-1 and human normal bronchial epithelial 16HBE cells were all purchased from the Institute of Biochemistry and Cell Biology of the Chinese Academy of Sciences (Shanghai, China). These cells were cultured in RPMI-1640 medium (Thermo Fisher Scientific, Inc., Waltham, MA, USA) supplemented with $10 \%$ fetal bovine serum (FBS; Gibco; Thermo Fisher Scientific, Inc.), 100 IU/ml penicillin and $100 \mathrm{IU} / \mathrm{ml}$ streptomycin (Gibco; Thermo Fisher Scientific, Inc.) in an incubator at $37^{\circ} \mathrm{C}$ with $5 \% \mathrm{CO}_{2}$.

miR-375mimictransfection. Human NSCLC cells were cultured in RPMI-1640 supplemented with $10 \%$ FBS. After $24 \mathrm{~h}$ the cells were transfected with mimic (miR-375 or control; Thermo Fisher Scientific, Inc.) using Lipofectamine 2000 (Invitrogen; Thermo Fisher Scientific, Inc.). The final concentration of the mimic was $10 \mathrm{nM}$.

Reverse transcription-quantitative polymerase chain reaction $(R T-q P C R)$. The total RNA was extracted from human NSCLC cells using TRIzol reagent (Invitrogen; Thermo Fisher Scientific, Inc.) and the RNA was reverse transcribed using a TaqMan miRNA Reverse Transcription kit (Applied Biosystems; Thermo Fisher Scientific, Inc.) with an miRNA-specific looped RT primer (Applied Biosystems; Thermo Fisher Scientific, Inc.) following the manufacturer's protocol. The RT-qPCR of miR-375 was performed using TaqMan Universal PCR Master mix with miRNA-specific TaqMan minor groove binder probes, purchased from Applied Biosystems (Thermo Fisher Scientific, Inc.). RNA U6 was used as an internal control. RT-qPCR was used to determine the expression of miR-375 using primers obtained from Thermo Fisher Scientific, Inc under the following thermocycling conditions: $95^{\circ} \mathrm{C}$ for $20 \mathrm{sec}$, followed by 40 cycles at $95^{\circ} \mathrm{C}$ for $10 \mathrm{sec}$ and at $60^{\circ} \mathrm{C}$ for $20 \mathrm{sec}$. Relative expression was quanitifed using the $2^{-\Delta \Delta \mathrm{Cq}}$ method (18).
RT-qPCR was also used to determine the expression of HER-2. The following thermocycling conditions were used: $95^{\circ} \mathrm{C}$ for $10 \mathrm{~min}$, followed by 40 cycles of $15 \mathrm{sec}$ at $95^{\circ} \mathrm{C}$ and $30 \mathrm{sec}$ at $60^{\circ} \mathrm{C}$. The primers used were as follows: HER-2, forward (F) 5'-CCAGCCTTCGACAACCTCTATT-3', reverse (R) 5'-TGCCGTAGGTGTCCCTTTG-3'; probe 5'-FAM-ACC AGGACCCACCAGAGCGGG-TAMRA-3'; $\beta$-actin, F 5'-ATC TGGCACCACACCTTCTACAAT-3', R 5'-CCGTCACCG GAGTCCATCA-3', probe 5'-FAM-TGACCCAGATCATGT TTGAGACCTTCAACAC-TAMRA-3'. The RT-qPCR experiments were performed in triplicate and the data were presented as the mean \pm standard deviation.

Cell Counting kit-8 (CCK-8) assay. Cell proliferation was determined using CCK-8 assay. Human NSCLC cells were seeded into 96 -well plates at a density of $5 \times 10^{3}$. The cells were cultured in incubator at $37^{\circ} \mathrm{C}$ with $5 \% \mathrm{CO}_{2}$. At $2 \mathrm{~h}$ prior the end point, CCK-8 reagent (1:10) was added to the wells and the cells were incubated for an additional $2 \mathrm{~h}$ at $37^{\circ} \mathrm{C}$. The absorbance of each well was also determined and was expressed as the proliferation of the cells. The studies were performed in triplicate and the data are presented as the mean \pm standard deviation.

Colony formation assay. A total of 550 human NSCLC cells were cultured in 6-well plates in RPMI-1640 medium for 2 weeks. The colonies were fixed with methanol and stained with $0.1 \%$ crystal violet in $20 \%$ methanol. The number of colonies were counted by using a microscope. All the studies were performed in triplicate and the data are presented as the mean \pm standard deviation.

Flow-cytometric analysis of apoptosis. Human NSCLC cells were cultured for $48 \mathrm{~h}$ and harvested. The cells were stained with annexin $\mathrm{V}$ and propidium iodide (Beyotime Institute of Biotechnology, Haimen, China) following the manufacturer's protocol. The apoptosis assay was performed using flow cytometry (BD Biosciences, Franklin Lakes, NJ, USA) and quantified with CellQuest software (BD Biosciences). The studies were performed in triplicate, and the data are presented as the mean \pm standard deviation.

Western blotting. Proteins were extracted with RIPA lysis buffer (Beyotime Institute of Biotechnology). Protein concentration was detected using a BCA protein assay kit (Beyotime Institute of Biotechnology). A total of $40 \mu \mathrm{g}$ protein per lane was separated by $15 \%$ SDS PAGE gel and was subsequently transferred onto a polyvinylidene difluoride membrane. The membranes were blocked with 5\% non-fat dried milk for $2 \mathrm{~h}$ at room temperature. Following blocking, the membranes were incubated with HER-2 primary antibody (cat. no. 2242; 1:1,000; Cell Signaling Technology, Inc., Danvers, MA, USA) for $2 \mathrm{~h}$. The membranes were washed with TBST three times and were subsequentlly incubated with horseradish peroxidase-conjugated secondary antibody (cat. no. A0208; 1:1,000; Beyotime Institute of Biotechnology) for an additional $2 \mathrm{~h}$. The membranes were washed and the proteins were visualized using enhanced chemiluminescence (Beyotime Institute of Biotechnology) and exposed on x-ray film. All experiments were performed in triplicate. 
Dual-luciferase assay. The full length of HER-2 was cloned into the downstream region of the firefly luciferase gene using the pGL3-control vector and the pMIR REPORT system (Thermo Fisher Scientific, Inc.). Mutant HER-2 was used as the corresponding control. The HER-2 report vectors were co-transfected with the mimics into A549 cells ata density of $1 \times 10^{4}$ using Lipofectamine 2000. Transfected cells were cultured for $48 \mathrm{~h}$, and were subsequently harvested for dual-luciferase assay (Promega Corporation, Madison, WI, USA). The relative luciferase activity was normalized to Renilla luciferase activity. The experiments were performed in triplicate and the data were presented as the mean \pm standard deviation.

In vivo mouse experiments. To investigate the effect of miR-375 on human NSCLC cell migration and invasion ability in vivo, A549 human NSCLC cells ( $\sim 3$ million cells/mouse) were injected into mice via the tail vein (6 mice/group). The following treatment groups were used: i) Mock A549 group; ii) mimic-control group iii) miR-375 mimic group. The mice were sacrificed using $\mathrm{CO}_{2}$ after 28 days and lung tissues were harvested. The number of metastatic tumor nodules were counted. The lungs were subsequently injected with India ink and fixed in AAF solution (ethanol:glacial acetic acid:formalin=17:1:2, obtained from Yuanmu Biotechnology, Shanghai, China). The number of metastatic nodules in the lung tissue of the mice were counted using a dissection microscope. The experiments were performed in triplicate and data are presented as the mean \pm standard error of the mean.

Statistical analysis. The data are expressed as the mean \pm standard deviation/standard error of the mean. One-way analysis of variance was used to determine the significance of the differences between the different treatment groups using SPSS version 13.0 (SPSS, Inc., Chicago, IL, USA). P $<0.05$ was considered to indicate a statistically significant difference.

\section{Results}

miR-375 expression is downregulated in human NSCLC cell lines. A previous study indicated that the expression level of miR-375 was reduced in tissues obtained from patients with NSCLC (17). The present study used RT-qPCR to determine the expression of miR-375 in human NSCLC cell lines. A total of four NSCLC adenocarcinoma cell lines and one NSCLC squamous carcinomas cell line were used, and 16HBE cells were used as a normal human bronchial epithelial cell control. The RT-qPCR results indicated that the expression of miR-375 was significantly reduced in human NSCLC cells compared with the normal human bronchial epithelial cell 16HBE $(\mathrm{P}<0.001$; Fig. 1). Therefore, the reduction of miR-375 expression may contribute to the progression and development of human NSCLC. A549 and SK-MES-1 cells were selected for the further study as A549 is the most common NSCLC adenocarcinoma cell and SK MES 1 is only NSCLC squamous carcinoma cell line used in the present study.

Manipulation of miR-375 levels in NSCLC cells by mimic-miR-375 transfection assays. To selectively increase the expression of miR-375 in human NSCLC cells, an

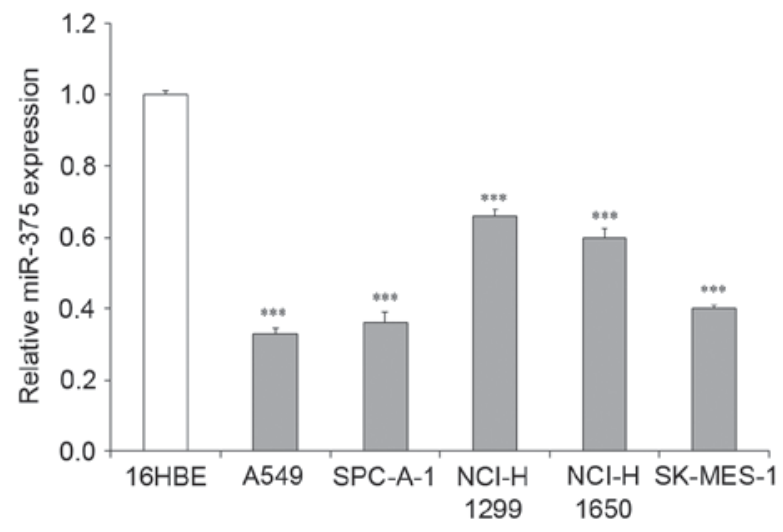

Figure 1. Expression of miR-375 in different human NSCLC cell lines. The data are presented as fold-changes in NSCLC cell lines compared with the normal bronchial epithelial cell line (16HBE). All experiments were performed in triplicate and the data were presented as the mean \pm standard deviation ${ }^{* * * *} \mathrm{P}<0.001$ vs.16HBE). NSCLC, non-small cell carcinoma; miR, mciroRNA.

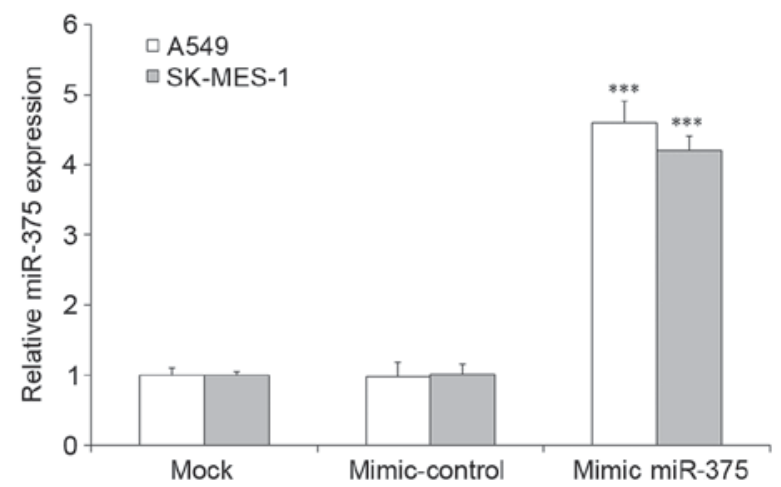

Figure 2. Expression of miR-375 in mock NSCLC cell lines and NSCLC cells transfected with mimic-miR375 or mimic-control. All experiments were performed in triplicate and the data are presented as the mean \pm standard deviation $\left({ }^{* * *} \mathrm{P}<0.001\right.$ vs. mock). NSCLC, non-small cell carcinoma; miR, microRNA.

miR-375 mimic transfection assay was used. A549 and SK-MES-1 cells were transfected with mimic-miR-375 or mimic-control, and were cultured. RT-qPCR was performed to determine the expression of miR-375 2 days after transfection. The results indicated that the expression of miR-375 was significantly increased following the transfection of mimic-miR-375 compared with the mimic control group, whereas the miR-375 expression in the mimic-control group was unaffected $(\mathrm{P}<0.001 ;$ Fig. 2$)$.

Overexpression of miR-375 inhibits NSCLC cell proliferation. In order to determine the effect of miR-375 in human NSCLC cell lines, the present study investigated the effects of overexpression of miR-375 on cell proliferation in vitro. A549 and SK-MES-1 human NSCLC cell lines were transfected with mimic-miR-375, mimic-control or mock, and were cultured for $96 \mathrm{~h}$. Cell proliferation was quantified every $24 \mathrm{~h}$ using the CCK- 8 assay. The cell proliferation results indicated that induction of miR-375 expression significantly reduced the proliferation of A549 and SK-MES-1 cells, whereas the mimic-control transfection failed to affect the cell proliferation 
A

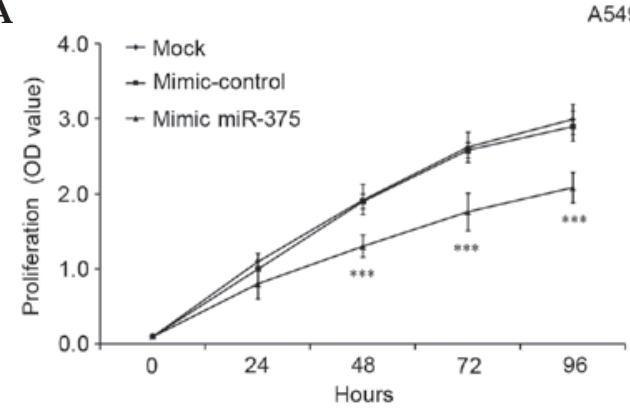

B

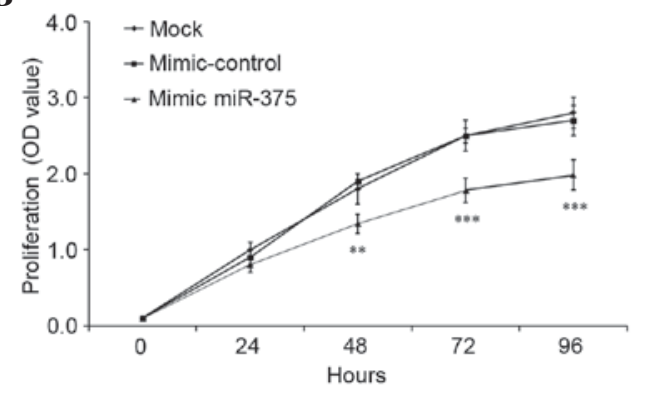

C

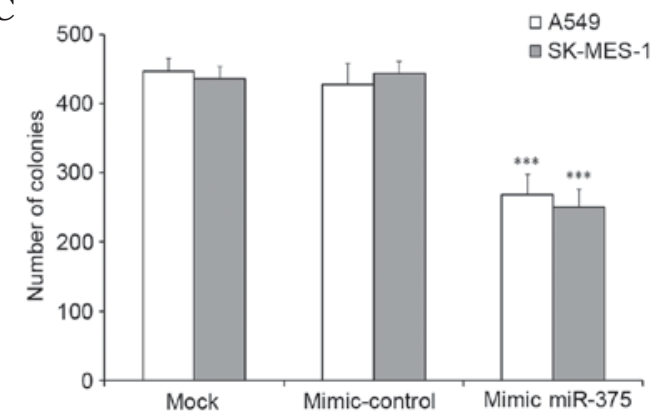

Figure 3. Effect of miR-375 expression on human non-small cell carcinoma cell survival. Cell proliferation of (A) A549 and (B) SK-MES-1 cell lines following transfection with miR-375, control or mock sequence. (C) Colony formation of A549 and SK-MES-1 cell lines following transfection with miR-375, control or mock sequence. All experiments were performed in triplicate and the data are presented as the mean \pm standard deviation. ${ }^{* *} \mathrm{P}<0.01$, ${ }^{* * *} \mathrm{P}<0.001$ vs. mock. OD, optical density; miR, microRNA.

compared with the mock cells ( $\mathrm{P}<0.001$; Fig. $3 \mathrm{~A}$ and $\mathrm{B})$. The colony-formation of the cells in the different treatment groups indicated that the number of colonies formed was significantly reduced in the mimic-miR-375-transfected group compared with the mock and mimic-control cells $(\mathrm{P}<0.001$; Fig. 3C).

Overexpression of miR-375 induces apoptosis of NSCLC cells. Wang et al (19) indicated that the inhibition of cell growth was due to the apoptosis of cancer cells (19). The present study aimed to identify whether the inhibition of NSCLC proliferation was due to the induction of apoptosis using flow cytometry. Mock and mimic-transfected A549/SK-MES-1 cells were cultured for $48 \mathrm{~h}$, and the cells were subsequently stained with annexin $\mathrm{V}$ and propidium iodide to assess cell apoptosis. The results demonstrated that the apoptotic rate of the cells transfected with mimic-miR-375 was significantly increased compared with the mock group ( $\mathrm{P}<0.001 ;$ Fig. 4). No difference was observed between the mock and the mimic-control groups (Fig. 4). Therefore, the upregulation of miR-375 may induce

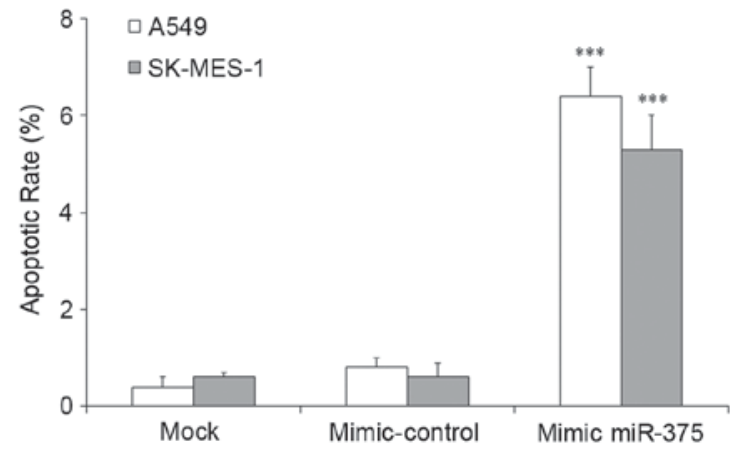

Figure 4. Flow cytometric analysis of cell apoptosis in A549 and SK-MES-1 cells transfected with mimic-miR375, mimic-control and mock cells. All experiments were performed in triplicate and the data are presented as the mean \pm standard deviation. ${ }^{* * *} \mathrm{P}<0.001$ vs. mock. miR, microRNA.

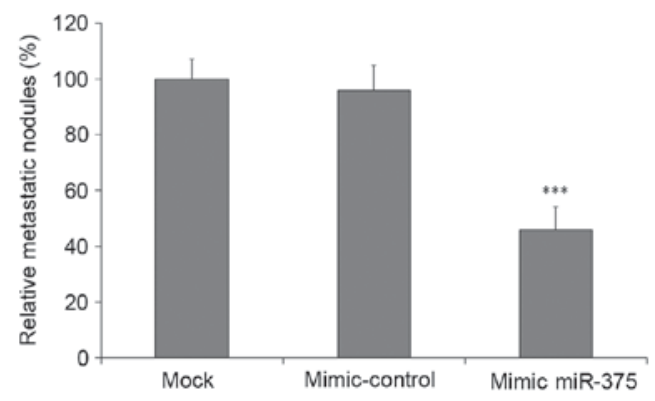

Figure 5. Effect of miR-375 transfection on the migration and invasion abilities of A549 cells in vivo. The cells were injected intravenously via the tail vein. Mice were sacrificed 4 weeks after the injection and the relative metastatic nodules were quantified. The data are presented as the mean \pm standard error of the mean. ${ }^{* * *} \mathrm{P}<0.001$ vs. mock. miR, microRNA.

the apoptosis of human NSCLC cells, which may subsequently lead to the inhibition of NSCLC cell proliferation.

Overexpression of miR-375 suppresses the migration and invasion of NSCLC cells in vivo. In order to investigate whether the induction of miR-375 may affect the migration and invasion abilities of NSCLC cells in vivo, A549 human NSCLC cells exposed to various treatments (mock, transfected with mimic-miR375 or mimic-control) were injected into nude mice intravenously via the tail vein. After 28 days the mice were sacrificed and the lung tissues were harvested. Large tumors were observed in the mock and mimic-control groups, whereas in mimic-miR-375 group large tumors were not evident (data not shown). The results indicated that the number of relative lung tumor nodules was significantly reduced in the mimic-miR-375 group compared with the mock and mimic-control group ( $\mathrm{P}<0.001$; Fig. 5). Therefore, upregulation of miR-375 may inhibit the migration and invasion of NSCLC cells in vivo and miR-375 may contribute to the reduction of the development of NSCLC.

HER-2 is a direct target of $m i R-375$. In order to identify the potential target of miR-375, the present study identified potential target genes using TargetScan (targetscan.org/vert_71/). It was determined that HER-2 was a potential target gene for miR-375 (Fig. 6A). In order to confirm whether 

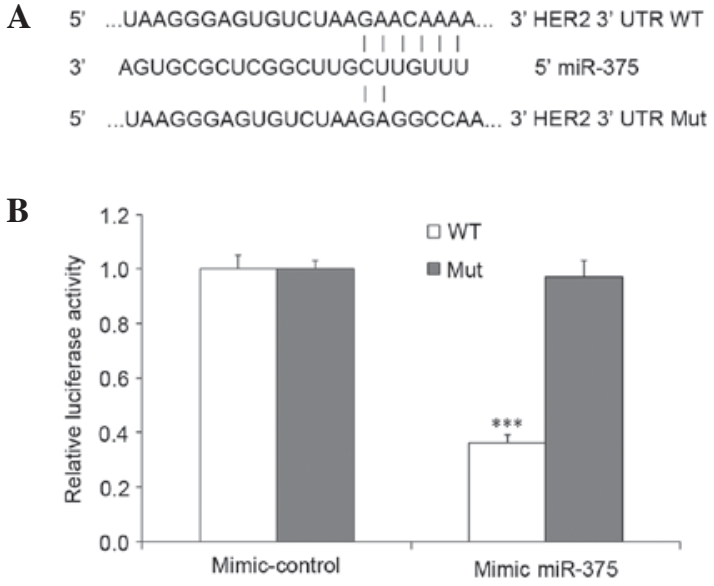

C

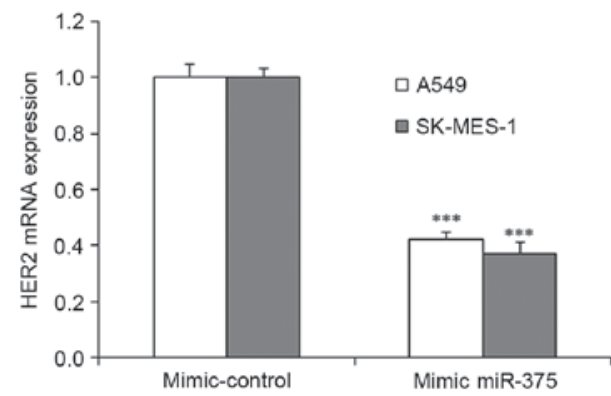

D

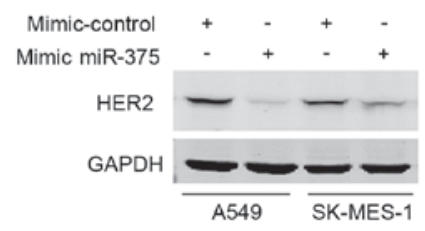

Figure 6. miR-375 regulated the human NSCLC cell survival by targeting HER2. (A) Prediction of the miR-375 binding sites in the 3'UTRs of human HER2 gene. (B) HER2 is a direct target gene of miR-375, as shown using a luciferase assay. (C) mRNA expression level of HER2 in human NSCLC cells with or without mimic-miR375 transfection. (D) Western blot assay was used to determine the protein level of HER2 in human NSCLC cells with or without mimic-miR375 transfection. All experiments were performed in triplicate and the data were presented as the mean \pm standard deviation. ${ }^{* * *} \mathrm{P}<0.001$ vs. mimic-control. NSCLC, non-small cell carcinoma; HER2, human epidermal growth factor receptor 2; WT, wild-type; Mut, mutant; UTR, untranslated region; miR, microRNA.

HER-2 was a direct target of miR-375, a dual luciferase assay was performed. A549 cells were co-transfected with mimic-miR-375 and pGL3-HER-2; mimic-control transfected cells were used as the control. The results indicated that in A549 cells transfected with mimic-miR-375 and pGL3-HER-2 wild-type, the luciferase activity was significantly reduced compared with the mimic control group ( $\mathrm{P}<0.001$; Fig. 6B). In A549 cells transfected with mimic-miR-375 and pGL3-HER-2 mutated, no reduction in luciferase activity was observed (Fig. 6B). Therefore, it was confirmed that HER-2 may be the direct target of miR-375. The expression level of HER-2 in mimic-miR-375 transfected A549/SK-MES-1 cells was also determined. It was revealed that the mRNA expression was significantly reduced following the transfection of miR-375 compared with the mimic-contro group $(\mathrm{P}<0.001$; Fig. $6 \mathrm{C})$. No changes were observed in the mimic-control group (Fig. 6C).
The protein expression of HER-2 was also reduced in the mimic-miR-375 group (Fig. 6D).

\section{Discussion}

Previous studies indicated that miRNAs may act as oncogenes or tumor suppressor genes, as they regulate the process of cancer development (12-16). miR-375 was initially identified in the pancreas (20), as it was highly expressed in the tissue of pancreatic islets and the brain (21). Previous studies revealed that miR-375 has been identified in various types of cancer, including breast and gastric cancer (22-24). Shen et al (25) revealed that miR-375 was abnormally expressed in gastric cancer cells and that increased the expression of miR-375 inhibited their proliferation (25). Zhang et al (26) determined that miR-375 inhibited the proliferation of mouse pancreatic progenitor cells by targeting Yes associated protein 1 (26). Recently, Li et al (17) reported that miR-375 was downregulated in human NSCLC tissues (17). However, to the best of our knowledge, the level of miR-375 in human NSCLC cell lines has not been investigated and the effect of miR-375 in human cells remains to be elucidated.

The present study determined that the expression of miR-375 in five different human NSCLC cell lines. The expression level of miR-375 in these cell lines was significantly reduced compared with the $16 \mathrm{HBE}$ normal human bronchial epithelial cell cells; this was in agreement with expression levels observed in tissues of patients with NSCLC. The abnormal regulation of miR-375 may be associated with a functional change in human NSCLC cells. Therefore, the biological activity of miR-375 in human NSCLC cells was investigated using mimic miR-375 transfection assay. It was determined that upregulation of miR-375 expression may inhibit the proliferation of human NSCLC cells. Additionally, it was identified that the inhibition may be due to induction of apoptosis in NSCLC cells. Animal models indicated that upregulation of miR-375 may reduce the migration and invasion ability of NSCLC cells in vivo. It was determined that the miR-375 expression was downregulated in all human NSCLC cell lines that were investigated in the present study. Upregulation of miR-375 in human NSCLC cells may inhibit the proliferation by increasing the apoptotic rate of human NSCLC cells, and inhibit their migration and invasion.

HER-2 has been identified as a member of the epidermal growth factor receptor family, and overexpression of HER-2 was also identified in human breast, which contributed to the development and progression of various types of cancer (25,27). Li et al (17) demonstrated that miR-375 expression is an important predictor of human NSCLC (17). A previous study indicated that miR-375 may inhibit the proliferation of gastric cancer cells by reducing HER-2 expression (25). The present study determined that HER-2 is a direct target gene of miR-375 and upregulation of miR-375 expression may reduce the mRNA and protein expression levels of HER-2. However, the association between cell apoptosis and reduced HER-2 expression remains to be elucidated, and further investigation of the molecular mechanisms is required.

In conclusion, the present study indicated that the expression of miR-375 was reduced in human NSCLC cell lines, and its upregulation may inhibit proliferation of NSCLC 
cells and induce apoptosis. It was also revealed that induction of miR-375 may inhibit the migration and invasion abilities of NSCLC cells in vivo. Therefore, miR-375 may be a potential therapeutic target for human NSCLC cancer.

\section{References}

1. Hoffman PC, Mauer AM and Vokes EE: Lung cancer. Lancet 355: 479-485, 2000

2. Verdecchia A, Francisci S, Brenner H, Gatta G, Micheli A, Mangone L and Kunkler I; EUROCARE-4 Working Group: Recent cancer survival in Europe: A 2000-02 period analysis of EUROCARE-4 data. Lancet Oncol 8: 784-796, 2007.

3. Liu XH, Lu KH, Wang KM, Sun M, Zhang EB, Yang JS, Yin DD, Liu ZL, Zhou J, Liu ZJ, et al: MicroRNA-196a promotes non-small cell lung cancer cell proliferation and invasion through targeting HOXA5. BMC Cancer 12: 348, 2012.

4. Ma Q, Jiang Q, Pu Q, Zhang X, Yang W, Wang Y, Ye S, Wu S, Zhong G, Ren J, et al: MicroRNA-143 inhibits migration and invasion of human non-small-cell lung cancer and its relative mechanism. Int J Biol Sci 9: 680-692, 2013.

5. Liu Y, Li M, Zhang G and Pang Z: MicroRNA-10b overexpression promotes non-small cell lung cancer cell proliferation and invasion. Eur J Med Res 18: 41, 2013.

6. Chi SW, Zang JB, Mele A and Darnell RB: Argonaute HITS-CLIP decodes microRNA-mRNA interaction maps. Nature 460: 479-486, 2009.

7. Hale BJ, Yang CX and Ross JW: Small RNA regulation of reproductive function. Mol Reprod Dev 81: 148-159, 2014.

8. Farazi TA, Spitzer JI, Morozov P and Tuschl T: miRNAs in human cancer. J Pathol 223: 102-115, 2011.

9. Bartel DP: MicroRNAs: Genomics, biogenesis, mechanism, and function. Cell 116: 281-297, 2004.

10. Calin GA and Croce CM: MicroRNA signatures in human cancers. Nat Rev Cancer 6: 857-866, 2006.

11. Ding J, Huang S, Wu S, Zhao Y, Liang L, Yan M, Ge C, Yao J, Chen T, Wan D, et al: Gain of miR-151 on chromosome 8q24.3 facilitates tumour cell migration and spreading through downregulating RhoGDIA. Nat Cell Biol 12: 390-399, 2010.

12. Ma L, Teruya-Feldstein J and Weinberg RA: Tumour invasion and metastasis initiated by microRNA-10b in breast cancer. Nature 449: 682-688, 2007.

13. Ma L, Reinhardt F, Pan E, Soutschek J, Bhat B, Marcusson EG, Teruya-Feldstein J, Bell GW and Weinberg RA: Therapeutic silencing of mir-10b inhibits metastasis in a mouse mammary tumor model. Nat Biotechnol 28: 341-347, 2010.
14. Cho WC: MicroRNAs: Potential biomarkers for cancer diagnosis, prognosis and targets for therapy. Int J Biochem Cell Biol 42: 1273-1281, 2010.

15. Cho WC: MicroRNAs in cancer-from research to therapy. Biochim Biophys Acta 1805: 209-217, 2010.

16. Gabriely G, Teplyuk NM and Krichevsky AM: Context effect: microRNA-10b in cancer cell proliferation, spread and death. Autophagy 7: 1384-1386, 2011.

17. Li Y, Jiang Q, Xia N, Yang H and Hu C: Decreased expression of microRNA-375 in nonsmall cell lung cancer and its clinical significance. J Int Med Res 40: 1662-1669, 2012.

18. Livak KJ and Schmittgen TD: Analysis of relative gene expression data using real-time quantitative PCR and the 2(-Delta Delta C(T)) method. Methods 25: 402-408, 2001.

19. Wang Y and Lee CG: MicroRNA and cancer-focus on apoptosis. J Cell Mol Med 13: 12-23, 2009.

20. Poy MN, Eliasson L, Krutzfeldt J, Kuwajima S, Ma X, Macdonald PE, Pfeffer S, Tuschl T, Rajewsky N, Rorsman P and Stoffel M: A pancreatic islet-specific microRNA regulates insulin secretion. Nature 432: 226-230, 2004.

21. Avnit-Sagi T, Kantorovich L, Kredo-Russo S, Hornstein E and Walker MD: The promoter of the pri-miR-375 gene directs expression selectively to the endocrine pancreas. PLoS One 4: e5033, 2009

22. Liu AM, Poon RT and Luk JM: MicroRNA-375 targets Hippo-signaling effector YAP in liver cancer and inhibits tumor properties. Biochem Biophys Res Commun 394: 623-627, 2010.

23. Poy MN, Hausser J, Trajkovski M, Braun M, Collins S, Rorsman P, Zavolan $M$ and Stoffel M: miR-375 maintains normal pancreatic alpha- and beta-cell mass. Proc Natl Acad Sci USA 106: 5813-5818, 2009.

24. Kloosterman WP, Lagendijk AK, Ketting RF, Moulton JD and Plasterk RH: Targeted inhibition of miRNA maturation with morpholinos reveals a role for miR-375 in pancreatic islet development. PLoS Biol 5: e203, 2007.

25. Shen ZY, Zhang ZZ, Liu H, Zhao EH and Cao H: miR-375 inhibits the proliferation of gastric cancer cells by repressing ERBB2 expression. Exp Ther Med 7: 1757-1761, 2014.

26. Zhang ZW, Men T, Feng RC, Li YC, Zhou D and Teng CB: miR-375 inhibits proliferation of mouse pancreatic progenitor cells by targeting YAP1. Cell Physiol Biochem 32: 1808-1817, 2013.

27. Mitri Z, Constantine T and O'Regan R: The HER2 receptor in breast cancer: Pathophysiology, clinical use and new advances in therapy. Chemother Res Pract 2012: 743193, 2012. 